\title{
Caracterização e comportamento higroscópico do pó da polpa de cajá liofilizada
}

\author{
Gleison S. Oliveira ${ }^{1}$, José M. C. da $\operatorname{Costa}^{2} \&$ Marcos R. A. Afonso ${ }^{3}$ \\ ${ }^{1}$ IFRN, Pau dos Ferros, RN. E-mail: gleison.silva@ifrn.edu.br \\ ${ }^{2}$ UFC, Fortaleza, CE. E-mail: correia@ufc.br \\ ${ }^{3}$ UFC, Fortaleza, CE. E-mail: m.r.a.afonso@gmail.com (Autor correspondente)
}

Palavras-chave:

alimentos em pó

isotermas

desidratação

Spondias mombin L.

\begin{abstract}
R E S U M O
A higroscopicidade de um alimento desidratado está ligada à sua estabilidade física, química e microbiológica. Este trabalho teve como objetivos a caracterização físico-química e o comportamento higroscópico dos pós da polpa de cajá liofilizada contendo maltodextrina (DE20). Foram feitas as análises de umidade, pH, sólidos solúveis, acidez total titulável, ácido ascórbico, açúcares, cor, higroscopicidade e o grau de caking. Para obtenção das isotermas os modelos de BET, GAB, Henderson e Oswin na temperatura de $25^{\circ} \mathrm{C}$ foram ajustados e avaliados. A adição de maltodextrina à polpa de cajá resultou em um pó com menor higroscopicidade e grau de caking. Além disto, os resultados demonstraram que a adição da maltodextrina confere um pó estatisticamente diferente $(\mathrm{p}<0,05)$ do pó sem adição de maltodextrina com relação ao teor de umidade, sólidos solúveis, acidez total titulável, ácido ascórbico, açúcares e cor. As isotermas dos pós apresentaram comportamento do tipo III, característico de materiais ricos em carboidratos. Os resultados mostraram que os melhores ajustes para as isotermas foram os modelos de BET e Henderson para o pó da polpa de cajá integral e o pó adicionado de maltodextrina, respectivamente.
\end{abstract}

Key words: food powder isotherms dehydration Spondias mombin L.

\section{Characterization and hygroscopic behavior of lyophilized yellow mombin in pulp powder}

\begin{abstract}
A B S T R A C T
The hygroscopicity of the dehydrated food is related to its physical, chemical and microbiological stability. This study aimed to characterize the physico-chemical and hygroscopic behavior of freeze dried yellow mombin pulp powder with maltodextrin (DE20). Analysis of moisture, $\mathrm{pH}$, soluble solids, titratable acidity, ascorbic acid, sugars, color, hygroscopicity and caking degree were performed. To obtain the isotherm, BET, GAB, Henderson and Oswin models were fitted and evaluated at $25^{\circ} \mathrm{C}$. The addition of maltodextrin to yellow mombin pulp resulted in a powder with a lower hygroscopicity and degree of caking. In addition, the results showed that the addition of maltodextrin provides a powder statistically different $(p<0.05)$ than the powder without maltodextrin with respect to moisture content, soluble solids, titratable acidity, ascorbic acid, sugars and color. The isotherms of the powders were classified as type III, characteristic of materials rich in carbohydrates. The results showed that the best fit of the isotherms were BET and Henderson models for the yellow mombin pulp powder and the yellow mombin pulp powder with maltodextrin, respectively.
\end{abstract}

\section{INTRODUÇÃO}

O cajá (Spondias mombin L.) é um fruto bastante apreciado em diversas regiões do País devido ao seu aroma e seu sabor agradável e exótico, aliados às boas características para a industrialização. No Brasil, a cajazeira é encontrada principalmente nos Estados do Norte e Nordeste cujos frutos recebem diferentes denominações, tais como, cajá, cajá verdadeiro, cajá-mirim e taperebá (Soares et al., 2006). É utilizado na fabricação de sucos, sorvetes, geleias, polpas congeladas e consumo in natura despertando o interesse não apenas para o mercado regional mas também para outros locais do país.

Embora o cajá tenha tido expansão e desenvolvimento de seu cultivo nos últimos anos, por ser um fruto muito perecível e ter sua produção entre 3 a 4 meses por ano, seu consumo in natura é muito limitado (Mata et al., 2005). Desta forma é necessária uma tecnologia de processamento para conservação a fim de disponibilizá-lo por maiores períodos.

A desidratação de frutos é um processo que agrega valor ao produto e diversifica sua utilização. Uma das propriedades que devem ser levadas em consideração nos frutos desidratados é sua higroscopicidade. Alimentos em pó com altas higroscopicidades promovem um fenômeno conhecido como caking, que dificulta a utilização desses produtos (Carlos et al., 2005). A higroscopicidade de um alimento está ligada à sua estabilidade física, química e microbiológica; desta forma, torna-se imprescindível o conhecimento do comportamento higroscópico desses produtos (Oliveira et al., 2012). 
Alimentos que contenham muitos açúcares, tais como sacarose, glicose e frutose são, geralmente, muito higroscópicos. Esses açúcares são responsáveis por fortes interações com moléculas de água principalmente quando se apresentam no estado amorfo (Jaya \& Das, 2004; Carlos et al., 2005).

A liofilização de sucos de frutas produz pós com altas quantidades de açúcares amorfos sendo, portanto, responsável pela elevada higroscopicidade desses pós (Carlos et al., 2005). Adjuvantes de secagem podem ser misturados aos sucos ou polpas de frutas com a finalidade de reduzir a higroscopicidade dos pós obtidos. A maltodextrina é um adjuvante muito utilizado na obtençao de alimentos em pó, incluindo aqueles por liofilização. Maltodextrinas são obtidas pela hidrólise do amido constituídas de unidades $\beta$-D-glicose e classificadas de acordo com sua dextrose equivalente (DE) (Kurozawa et al., 2009)

Uma forma de se conhecer o comportamento higroscópico de alimentos em pó é através de suas isotermas de sorção. O conhecimento das isotermas de sorção de umidade dos alimentos tem aplicação na predição do tempo de secagem, vida útil do produto, determinação do tipo de embalagem e na caracterização do produto, inclusive quando o mesmo é constituído por componentes de atividade de água diferentes (Alexandre et al., 2007).

Inúmeros modelos matemáticos têm sido propostos para descrever o comportamento das isotermas de sorção de alimentos. Não há uma equação geral que descreva todas as isotermas dos alimentos visto que a atividade de água depende bastante da composição do alimento e da interação de seus diferentes constituintes com a água em condições de equilíbrio termodinâmico. Modelos matemáticos permitem que, com poucos pontos experimentais, seja possível a determinação das isotermas de sorção que podem ser extrapoladas para obtenção de pontos nas regiões de baixa ou alta atividade de água, pontos esses de difícil determinação.

O objetivo deste trabalho foi a obtenção das isotermas de adsorção de umidade para os pós da polpa de cajá integral liofilizada e adicionada de maltodextrina, além da caracterização físico-química dos pós.

\section{Material e Métodos}

As polpas de cajá foram adquiridas em indústria localizada na cidade de Fortaleza, CE, acondicionadas em embalagem de polietileno contendo $100 \mathrm{~g}$ de polpa. Permaneceram congeladas a $-18^{\circ} \mathrm{C}$ em freezer convencional até realização dos experimentos.

Para realização dos experimentos as amostras foram descongeladas, distribuídas em bandejas e previamente congeladas a $-38{ }^{\circ} \mathrm{C}$ durante $24 \mathrm{~h}$; a seguir, foram desidratadas durante $24 \mathrm{~h}$ em liofilizador da marca TERRONI, modelo LS3000. Nas desidratações foram utilizadas duas formulações: polpa de cajá integral e polpa de cajá adicionada de $17 \%\left(\mathrm{~m} \mathrm{~m}^{-1}\right)$ de maltodextrina dextrose equivalente (DE) 20. Os pós obtidos foram acondicionados em filme plástico de polietileno/nylon ao abrigo da luz.
Para a caracterização físico-química dos pós obtidos foram realizadas, em triplicata, as seguintes análises: sólidos solúveis ( ${ }^{B}$ Brix), acidez total em ácido cítrico, umidade e $\mathrm{pH}$, segundo a metodologia descrita pelo IAL (2008); análise de cor, com determinação no modo $\mathrm{CIE} \mathrm{L}^{\star} \mathrm{a}^{\star} \mathrm{b}^{*}$; açúcares solúveis totais e redutores determinados pelo princípio da redução do DNS; ácido ascórbico, conforme metodologia de Strohecker Henning (1967).

A higroscopicidade foi determinada segundo Goula \& Adamopoulos (2010) com modificações (solução saturada de $\mathrm{NaCl}$ e ganho de massa após $120 \mathrm{~min}$ ). O grau de caking dos pós foi determinado conforme Jaya \& Das (2004).

$\mathrm{Na}$ determinação das isotermas de adsorção dos pós foram pesados, em triplicata, 0,2 $\mathrm{g}$ de amostra dos pós em cadinhos de alumínio previamente tarados; posteriormente, os cadinhos contendo as amostras foram colocados em células fechadas contendo soluções salinas a fim de condicionar o ambiente interno com valores de umidade relativa, de acordo com Greenspan (1977) (Tabela 1). A temperatura utilizada na determinação das isotermas foi de $25,0 \pm 2,0{ }^{\circ} \mathrm{C}$.

Tabela 1. Umidades relativas das células a $25 \pm 2{ }^{\circ} \mathrm{C}$ de acordo com soluções salinas saturadas

\begin{tabular}{ccc}
\hline Célula & Soluções saturadas & Umidade relativa (\%) \\
1 & $\mathrm{CH}_{3} \mathrm{COOK}$ & 21 \\
2 & $\mathrm{~K}_{2} \mathrm{CO}_{3}$ & 44 \\
3 & $\mathrm{NaBr}$ & 58 \\
4 & $\mathrm{SnCl}_{2}$ & 76 \\
5 & $\mathrm{KCl}$ & 84 \\
6 & $\mathrm{BaCl}_{2}$ & 90 \\
\hline
\end{tabular}

As amostras de cada célula foram pesadas em balança analítica em intervalos regulares de $24 \mathrm{~h}$ até a obtenção do equilíbrio, ou seja, quando não houve mais variação de massa. Alcançado o equilíbrio foi determinada a atividade de água das amostras a $25^{\circ} \mathrm{C}$, utilizando-se um medidor de atividade de água (AQUALab, modelo 4TEV); a seguir, as amostras foram levadas para estufa a vácuo com temperatura de $70{ }^{\circ} \mathrm{C}$ para determinação das massas secas.

A umidade de equilíbrio de cada amostra $\left(\mathrm{X}_{\mathrm{eq}}\right)$ foi calculada segundo a Eq. 1 .

$$
\mathrm{X}_{\mathrm{eq}}=\frac{\mathrm{m}_{\mathrm{o}}-\mathrm{m}_{\mathrm{s}}}{\mathrm{m}_{\mathrm{s}}}
$$

em que:

$$
\begin{array}{ll}
\mathrm{X}_{\mathrm{eq}} & \text { - umidade de equilíbrio, } \mathrm{g} \mathrm{g}^{-1} \\
\mathrm{~m}_{0} & \text { - massa da amostra no equilíbrio, } \mathrm{g} \\
\mathrm{m}_{\mathrm{s}} & \text { - massa da amostra seca, } \mathrm{g}
\end{array}
$$

Para o ajuste matemático dos dados experimentais para obtenção das isotermas de adsorção foram utilizados os modelos matemáticos apresentados na Tabela 2 .

Para os ajustes foi utilizado o software Statistica versão 7.0. Nas avaliações dos modelos ajustados foram utilizados o coeficiente de determinação $\left(\mathrm{R}^{2}\right)$ e o resíduo médio relativo (E) (Eq. 6). 
Tabela 2. Modelos matemáticos utilizados para o ajuste das isotermas de adsorção do pó da polpa de cajá liofilizada

\begin{tabular}{cc}
\hline Modelos & $X_{\text {eq }}=\frac{\text { Equações* }^{*}}{\left(1-K \cdot a_{w}\right) \cdot\left(1-K \cdot a_{w}+C \cdot K \cdot a_{w}\right)}$ \\
GET & $X_{\text {eq }}=\frac{X_{m} \cdot C \cdot a_{w}}{\left(1-a_{w}\right)} \cdot\left[\frac{1-(n+1) \cdot\left(a_{w}\right)^{n}+n \cdot\left(a_{w}\right)^{n+1}}{1-(1-C) \cdot a_{w}-C \cdot\left(a_{w}\right)^{n+1}}\right]$ \\
Henderson & $X_{\text {eq }}=\left[\frac{-\ln \left(1-a_{w}\right)}{b}\right]^{\frac{1}{a}}$ \\
Oswin & $X_{\text {eq }}=a \cdot\left[\frac{a_{w}}{1-a_{w}}\right]^{b}$ \\
\hline
\end{tabular}

${ }^{*} \mathrm{X}_{\text {eq }}$ - Umidade de equilíbrio $\left(\mathrm{g} \mathrm{H}_{2} \mathrm{O} . \mathrm{g}^{-1}\right) ; \mathrm{X}_{\mathrm{m}}$ - Conteúdo de água na monocamada molecular $(\mathrm{g}$ $\left.\mathrm{H}_{2} \mathrm{O} . \mathrm{g}^{-1}\right) ; \mathrm{a}_{\mathrm{w}}$ - Atividade de água; $\mathrm{n}$ - número de camadas moleculares; $\mathrm{C}, \mathrm{K}$ - Constantes de sorção; a, b - parâmetros de ajuste

$$
E=\frac{100}{n} \sum_{i=1}^{n} \frac{\left|\left(M_{i}-M_{p}\right)\right|}{M_{i}}
$$

em que:

$\begin{array}{ll}E & \text { - resíduo médio relativo, \% } \\ M_{i} & \text { - valor experimental } \\ M_{p} & \text { - valor predito pelo modelo } \\ n & \text { - número de dados experimentais. }\end{array}$

\section{Resultados e Discussão}

Na Tabela 3 são apresentados os resultados dos parâmetros físico-químicos dos pós obtidos nas secagens por liofilização.

$\mathrm{O} \mathrm{pH}$ das duas amostras analisadas não apresentou diferença significativa ( $p>0,05$ ), variando de 2,65 a 2,63, tal como também os conteúdos de açúcares redutores, de 26,98 a 27,525\%; já o conteúdo de açúcares totais apresentou um ligeiro aumento no pó contendo maltodextrina (30,53\%) em comparação com o pó sem maltodextrina $(27,55 \%)$.

O efeito da adição de $17 \%$ de maltodextrina à polpa de cajá provocou uma drástica diminuição na acidez titulável e em outros componentes. A quantidade de sólidos totais na polpa de cajá está em torno de 10\% (Mata et al., 2005); ao se

Tabela 3. Valores médios da caracterização física e físico-química dos pós de cajá obtidos por liofilização

\begin{tabular}{lrr}
\hline \multicolumn{1}{c}{ Análise } & \multicolumn{1}{c}{ A } & \multicolumn{1}{c}{ B } \\
pH & $2,65^{\mathrm{a}} \pm 0,01$ & $2,63^{\mathrm{a}} \pm 0,02$ \\
Acidez total (\% ác. cítrico) & $9,11^{\mathrm{a}} \pm 0,16$ & $3,18^{\mathrm{b}} \pm 0,26$ \\
Sólidos solúveis ("Brix) & $78,00^{\mathrm{b}} \pm 1,00$ & $92,67^{\mathrm{a}} \pm 0,58$ \\
Umidade (\%) & $2,05^{\mathrm{a}} \pm 0,14$ & $0,65^{\mathrm{b}} \pm 0,17$ \\
Açúcares redutores (\%) & $26,98^{\mathrm{a}} \pm 0,25$ & $27,52^{\mathrm{a}} \pm 2,39$ \\
Aćúcares totais (\%) & $27,55^{\mathrm{b}} \pm 0,28$ & $30,53^{\mathrm{a}} \pm 1,41$ \\
Ácido ascórbico (mg 100 $\left.\mathrm{g}^{1}\right)$ & $168,47^{\mathrm{a}} \pm 1,63$ & $90,46^{\mathrm{b}} \pm 1,87$ \\
Higroscopicidade (\%) & $12,93^{\mathrm{a}} \pm 0,66$ & $8,51^{\mathrm{b}} \pm 0,40$ \\
Grau de caking (\%) & $33,65^{\mathrm{a}} \pm 3,52$ & $6,64^{\mathrm{b}} \pm 1,27$ \\
$\mathrm{~L}^{*}$ & $54,43^{\mathrm{b}} \pm 0,03$ & $58,41^{\mathrm{a}} \pm 0,20$ \\
$\mathrm{a}^{*}$ & $0,44^{\mathrm{a}} \pm 0,05$ & $-1,35^{\mathrm{b}} \pm 0,04$ \\
$\mathrm{~b}^{*}$ & $24,09^{\mathrm{a}} \pm 0,02$ & $21,34^{\mathrm{b}} \pm 0,02$ \\
\hline
\end{tabular}

A - Pó da polpa de cajá integral; B - Pó da polpa de cajá contendo 17\% (p/p) de maltodextrina; Médias seguidas do mesmo expoente na mesma linha, não diferem estatisticamente a nível de $5 \%$ pelo teste t-Student acrescentar $17 \%$ de maltodextrina à polpa, a quantidade deste agente no pó obtido chegou ao redor de $63 \%$ do total de sólidos. Explica-se, desta forma, a redução de $65 \%$ no teor da acidez no pó contendo maltodextrina (Tabela 3). Oliveira et al. (2006) também observaram, ao realizar uma análise comparativa entre a composição de polpa de pitanga integral e formulada com $15 \%$ $\left(\mathrm{m} \mathrm{m}^{-1}\right)$ de maltodextrina, redução na acidez total. Ferrari et al. (2012) constataram, comparando a adição de 5 e $25 \%\left(\mathrm{~m} \mathrm{~m}^{-1}\right)$ de maltodextrina ao suco de amora-preta, uma redução de 68,5\% no teor de antocianinas no pó do suco que continha a maior quantidade de maltodextrina. O conteúdo de ácido ascórbico entre as amostras diferiu estatisticamente entre si $(\mathrm{p} \leq 0,05)$. O pó contendo maltodextrina apresentou valor inferior $(90,46 \pm$ $\left.1,87 \mathrm{mg} 100 \mathrm{~g}^{-1}\right)$ ao pó da polpa integral $(168,47 \pm 1,63 \mathrm{mg} 100$ $\mathrm{g}^{-1}$ ) devido à adição da massa de maltodextrina à polpa; ainda assim, ambos os pós podem ser caracterizados como excelentes fontes desta vitamina $C$ visto que a ingestão diária recomendada (IDR) no Brasil, é de $45 \mathrm{mg}$ (Brasil, 2005). A quantidade de ácido ascórbico encontrada neste estudo é similar à do trabalho de Silva et al. (2005) que encontraram, em pó de umbu-cajá, o valor médio de $91,1 \mathrm{mg} 100 \mathrm{~g}^{-1}$.

A umidade dos pós diferiu estatisticamente entre si ( $p$ $\leq 0,05)$. O pó contendo maltodextrina apresentou menor umidade $(0,65 \pm 0,17 \%)$ que o pó integral $(2,05 \pm 0,15 \%)$; esta diminuição pode ser atribuída à maior quantidade de sólidos presentes.

Marques et al. (2006) obtiveram 7,06, 5,81 e 7,76\% de umidade para os pós de polpas liofilizadas de abacaxi, goiaba e manga, respectivamente. Esses valores foram superiores devido ao tempo de liofilização adotado por Marques et al. (2006) de apenas $12 \mathrm{~h}$ enquanto que o deste trabalho foi de $24 \mathrm{~h}$.

Nos valores da análise colorimétrica a luminosidade $\left(L^{\star}\right)$ do pó adicionado de maltodextrina apresentou maior valor devido a maltodextrina ser de cor branca, deixando a polpa mais clara. Quanto às coordenadas $\mathrm{a}^{*} \mathrm{e} \mathrm{b}^{*}$, observou-se uma diminuição nesses parâmetros no pó contendo maltodextrina. Esta redução da tonalidade do vermelho e amarelo também está relacionada à diluição da cor, provocada pela presença do agente carreador utilizado (Tonon et al., 2009).

A higroscopicidade dos pós diferiu significativamente ( $\mathrm{p}$ $\leq 0,05)$ entre os ensaios. No pó adicionado de maltodextrina $(8,51 \pm 0,4 \%)$ houve uma redução da higroscopicidade. Segundo Tonon et al. (2009) este comportamento se deve ao fato de a maltodextrina ser um material de baixa higroscopicidade e confirma a eficiência do seu uso como agente carreador no sentido de reduzir a higroscopicidade dos produtos desidratados.

$\mathrm{O}$ valor de grau de caking diferiu significativamente ( $\mathrm{p}$ $\leq 0,05)$ entre os dois ensaios apresentando menor valor no pó contendo maltodextrina $(6,64 \pm 1,27 \%)$. Os valores se encontram de acordo com o desejado para alimentos em pó, que variam de 5,0 a 34,0\%, como citam Jaya \& Das (2004). Goula \& Adamopoulos (2010) observaram, variando a quantidade de dextrose equivalente $(\mathrm{DE})$ da maltodextrina na atomização de suco de laranja concentrado, valores de grau de caking variando 
de 5,9 a 24,8\%; ainda segundo Goula \& Adamopoulos (2010) valores elevados para o grau de caking nos alimentos em pós são devidos à presença de açúcares.

Os parâmetros dos modelos matemáticos ajustados para as isotermas dos pós da polpa de cajá são apresentados na Tabela 4. Os modelos matemáticos utilizados obtiveram altos coeficientes de determinação $\left(\mathrm{R}^{2}\right)$ e baixos erros médios relativos $(\mathrm{E})$ para a maioria dos ajustes.

Com exceção dos modelos de BET e Oswin, na amostra do pó de cajá contendo maltodextrina, e Henderson, na amostra do pó de cajá integral, os demais modelos tiveram erro relativo abaixo de 10\%. Segundo Silva et al. (2005) pode-se considerar um modelo adequado para descrever um fenômeno em estudo quando este obtiver um erro relativo abaixo de $10 \%$, sendo este parâmetro utilizado para definir os modelos que melhor se adequam a este estudo. $\mathrm{O}$ modelo GAB foi o que melhor se ajustou às isotermas no trabalho de Bezerra et al. (2011) em pós das polpas de manga cv. Rosa e cv. Tommy Atkins. Jain et al. (2010) obtiveram melhores ajustes nos modelos Oswin e Henderson para as isotermas de mamão em cubos desidratado osmoticamente; já Alcântara et al. (2009) obtiveram, para as isotermas para o pedúnculo seco de caju, melhores ajustes pelos modelos de GAB seguidos pelo de BET.

Neste trabalho o modelo de BET forneceu o melhor ajuste para o pó da polpa de cajá pois neste modelo o valor de erro médio foi menor e apresentou maior coeficiente de determinação $\mathrm{R}^{2}$. De maneira similar, o modelo de Henderson foi o melhor para a representação da isoterma da amostra contendo maltodextrina. Estudando a atividade de água do cajá em pó microencapsulado com $15 \%$ de maltodextrina, Silva et al. (2005) citam o modelo de Henderson modificado por Cavalcanti-Mata como o modelo que melhor representou os dados experimentais para este produto.

Tabela 4. Parâmetros dos modelos matemáticos para representação das isotermas de sorção do pó de cajá a $25{ }^{\circ} \mathrm{C}$

\begin{tabular}{lccc}
\hline \multirow{2}{*}{ Modelos } & Parâmetros & \multicolumn{2}{c}{ Amostras } \\
\cline { 3 - 4 } GAB & $\mathrm{X}_{\mathrm{m}}$ & 0,09316 & $\mathbf{B}$ \\
& $\mathrm{C}$ & 3,70700 & 0,18010 \\
& $\mathrm{~K}$ & 0,99650 & 0,63830 \\
& $\mathrm{R}^{2}$ & 0,99890 & 0,83380 \\
& $\mathrm{E}(\%)$ & 4,93000 & 0,99850 \\
$\mathrm{BET}$ & $\mathrm{X}_{\mathrm{m}}$ & 0,08232 & 7,67100 \\
& $\mathrm{C}$ & 5,33000 & 0,10450 \\
& $\mathrm{n}$ & 1,29900 & 1,29300 \\
& $\mathrm{R}^{2}$ & 0,99900 & 1,45200 \\
Henderson & $\mathrm{E}(\%)$ & 4,53000 & 0,99480 \\
& $\mathrm{a}$ & 0,68600 & 13,75000 \\
& $\mathrm{~b}$ & 2,75400 & 0,74480 \\
& $\mathrm{R}^{2}$ & 0,99290 & 3,91700 \\
Oswin & $\mathrm{E}(\%)$ & 12,95000 & 0,99890 \\
& $\mathrm{a}$ & 0,14270 & 6,02200 \\
& $\mathrm{~b}$ & 0,80890 & 0,09734 \\
& $\mathrm{R}^{2}$ & 0,99820 & 0,81550 \\
& $\mathrm{E}(\%)$ & 5,79000 & 0,99550 \\
& & &
\end{tabular}

A - Pó da polpa de cajá integral; B - Pó da polpa de cajá contendo 17\% (p/p) de maltodextrina; $\mathrm{R}^{2}$ - Coeficiente de determinação; $\mathrm{E}$ - Erro médio relativo (\%); $\mathrm{X}_{\mathrm{m}}$ - Conteúdo de água na monocamada molecular $\left(\mathrm{g} \mathrm{H}_{2} \mathrm{O}_{\mathrm{g}} \mathrm{g}^{-1}\right) ; \mathrm{n}$ - Número de camadas moleculares; $\mathrm{C}, \mathrm{K}$ - Constantes de sorção; a, b - Parâmetros de ajuste
Segundo Pavan et al. (2012) os modelos de GAB e BET se baseiam no conceito de umidade na monocamada, sendo este um parâmetro importante para avaliação da estabilidade dos alimentos. $\mathrm{O}$ conteúdo de umidade na monocamada $\left(\mathrm{X}_{\mathrm{m}}\right)$ corresponde à quantidade de água fortemente adsorvida a sítios específicos na superfície dos alimentos e é considerado um valor crítico, acima do qual as taxas de algumas reações de degradação aumentam e a estabilidade da matriz alimentar diminui (Comunian et al., 2011). Os resultados do conteúdo de umidade na monocamada $\left(\mathrm{X}_{\mathrm{m}}\right)$ apresentados na Tabela 4 demonstram que a amostra contendo maltodextrina apresentou maiores valores em comparação com a amostra contendo apenas polpa de cajá. A amostra contendo maltodextrina mostrou-se mais estável, apresentando maiores valores de $\mathrm{X}_{\mathrm{m}}$ em ambos os modelos (GAB e BET) podendo ser exposta a ambientes com maiores umidades relativas se comparada à amostra do pó da polpa de cajá integral. Oliveira et al. (2012) também observaram, no estudo do comportamento das isotermas do pó de macaúba, que a adição de $8 \%\left(\mathrm{~m} \mathrm{~m}^{-1}\right)$ de maltodextrina elevou o valor do conteúdo de umidade da monocamada.

$\mathrm{O}$ valor da constante $\mathrm{K}$ de $\mathrm{GAB}$ representa uma medida das interações entre as moléculas do adsorvato com o adsorvente (Catelam et al., 2011). Segundo Timmermann (2003) a constante $\mathrm{K}$ de $\mathrm{GAB}$ aumenta com a força de interação entre adsorvato e adsorvente. Observa-se que, de acordo com a Tabela 4, a força de interação entre o vapor de água e a matriz sólida do pó da polpa de cajá diminui com a adição da maltodextrina, observando-se maior valor de $\mathrm{K}$ na amostra sem maltodextrina. De acordo com Chirife et al. (1992) valores de K não devem ser superiores a 1,0 pois tal comportamento é fisicamente inadequado. Neste trabalho os valores deste parâmetro foram inferiores aos da unidade e semelhantes aos reportados por Alexandre et al. (2007) no estudo das isotermas de adsorção do pó de pitanga.

Com relação aos parâmetros dos modelos de Henderson, o valor de 'a' deve ser maior que zero e 'b' maior que 1,0 e para o modelo de Oswin 'a' deve ser maior que zero e 'b' estar entre zero e 1,0 (Alcântara et al., 2009). Os valores dos parâmetros de Henderson e Oswin obtidos neste estudo se encontram dentro dessas limitações (Tabela 4).

As isotermas de adsorção de umidade para o pó da polpa de cajá com e sem adição de maltodextrina são apresentadas na Figura 1. Em ambas as isotermas um aumento da atividade de água representa aumento do conteúdo de água dos pós da polpa de cajá. As isotermas apresentaram forma característica do tipo III, de acordo com a classificação da IUPAC (1985). As mesmas formas de isotermas foram obtidas por Silva et al. (2008) estudando o comportamento higroscópico do açaí e cupuaçu em pó justificando que este comportamento é característico de materiais ricos em carboidratos. O mesmo comportamento foi observado por Alexandre et al. (2007) e Bezerra et al. (2011).

Observa-se, em ambas as isotermas (Figura 1) uma região inicial plana na qual grandes variações da atividade de água representam pouca alteração na umidade do pó porém a partir de atividade de água ao redor de 0,7 na isoterma do pó da polpa de cajá integral, este comportamento se altera, ou seja, qualquer pequeno aumento da atividade de água representa 


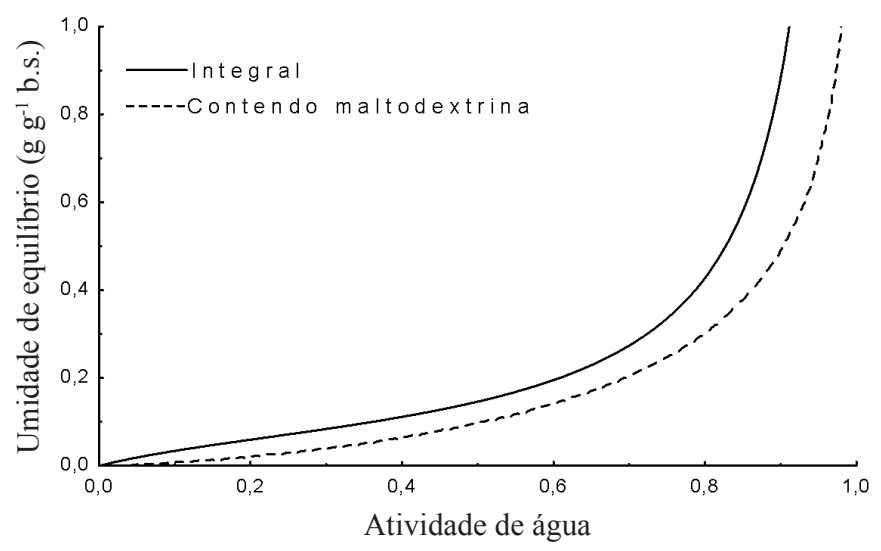

Figura 1. Isotermas de adsorção do pó da polpa de cajá segundo modelo de BET (polpa integral) e Henderson (polpa contendo maltodextrina, $17 \% \mathrm{~m} \mathrm{~m}^{-1}$ ) a $25^{\circ} \mathrm{C}$

um grande ganho de água pelo pó. O mesmo comportamento é observado na isoterma do pó contendo maltodextrina porém apenas a partir da atividade de água ao redor de 0,8 . Similar a este trabalho Bezerra et al. (2011) e Canuto et al. (2014) observaram um aumento exponencial da umidade de equilíbrio com pequenas variações da atividade de água, principalmente em valores acima de 0,7 ; deve-se, então, ter cuidado no armazenamento desses produtos em ambientes com umidade relativa acima de $70 \%$.

Observa-se, na Figura 1, que para um mesmo valor de atividade de água são observados valores menores de umidade de equilíbrio na isoterma do pó contendo maltodextrina. Similar a este trabalho, Mosquera et al. (2010) obtiveram, estudando o comportamento das isotermas do pó de borojó liofizado, para uma mesma atividade de água, menores valores de umidade de equilíbrio para as amostras que continham maltodextrina (DE 4,0-7,0 ou DE 16,5-19,5). Ainda segundo Mosquera et al. (2010) a presença da maltodextrina resultou em melhor estabilidade, menor higroscopicidade e aumento da temperatura de transição vítrea do pó de borojó. Ao estudar o comportamento das isotermas do pó da polpa de mamão liofilizada, Canuto et al. (2014) observaram que os valores de umidade de equilíbrio decresceram com o aumento da concentração de maltodextrina ao pó, demonstrando que a adição deste adjuvante reduz a higroscopicidade proporcionalmente à sua concentração. Wang \&Zhou (2012) também atribuem a redução da higroscopicidade do pó de molho de soja à adição de maltodextrina ao produto.

\section{Conclusões}

1. Os pós da polpa de cajá são boas fontes de ácido ascórbico e açúcares redutores, apresentam baixa higroscopicidade e baixo grau de caking.

2. A maltodextrina adicionada à polpa de cajá foi efetiva para diminuição da higroscopicidade, do grau de caking e o aumento do conteúdo de água na monocamada, gerando um pó mais estável.

3. O modelo de BET foi o que melhor se ajustou ao pó da polpa de cajá integral e o modelo de Henderson ao pó da polpa de cajá contendo maltodextrina DE20.
4. Apesar da adição da maltodextrina deslocar a isoterma do pó da polpa de cajá para região mais estável, o pó não deve ser exposto a ambientes com elevadas umidades relativas, sendo recomendado o uso de embalagens impermeáveis ao vapor de água.

\section{Literatura Citada}

Alcântara, S. R.; Almeida, F. A. C.; Silva, F. L. H.; Gomes, J. P. Isotermas de adsorção do pedúnculo seco do caju. Revista Brasileira de Engenharia Agrícola e Ambiental. v.13, p.8187, 2009

Alexandre, H. V.; Figueiredo, R. M. F.; Queiroz, A.J. de M. Isotermas de adsorção de umidade da pitanga em pó. Revista de Biologia e Ciências da Terra, v.7, p.11-20, 2007.

Bezerra, T. S.; Costa, J.M.C.; Afonso, M.R.A.; Maia, G.A.; Clemente, E. Avaliação físico-química e aplicação de modelos matemáticos na predição do comportamento de polpas de manga desidratadas em pó. Revista Ceres, v.58, p.278-283, 2011.

Brasil. Agência Nacional de Vigilância Sanitária. Resolução RDC n. 269, de 22 de setembro de 2005, que dispõe sobre o Regulamento Técnico sobre a ingestão diária recomendada (IDR) de proteína, vitaminas e minerais, Diário Oficial da República Federativa do Brasil, Brasília, DF, 2005.

Canuto, H. M. P.; Afonso, M. R. A.; Costa, J. M. C. Hygroscopic behavior of freeze-dried papaya pulp powder with maltodextrin. Acta Scientiarum Technology, v.36, n.1, 179-185, 2014.

Carlos, L. A.; Resende, J. V.; Cal-Vidal, J. Redução da higroscopicidade de pós liofilizados pela indução da cristalização em soluções-modelo de açúcares constituintes de frutas. Brazilian Journal of Food Technology, v.8, p.163173, 2005.

Catelam, K. T.; Trindade, C. S. F.; Romero, J. T. Water adsorption isotherms and isosteric sorption heat of spray-dried and freeze-dried dehydrated passion fruit pulp with additives and skimmed milk. Ciência e Agrotecnologia, v.35, p.11961203, 2011.

Chirife, J.; Timmermann, E. O.; Iglesias, H. A.; Boquet, R. Some features of the parameter $k$ of the GAB equation as applied to sorption isotherms of selected food products. Journal of Food Engineering, v.15, p.75-82, 1992.

Comunian, T.A.; Monterrey-Quintero, E. S.; Thomazini, M.; Balieiro, J. C. C.; Piccone, P.; Pittia, P.; Favaro-Trindade, C. S. Assessment of production efficiency, physicochemical properties and storage stability of spray-dried chlorophyllide, a natural food colourant, using gum Arabic, maltodextrin and soy protein isolate-based carrier systems. International Journal of Food Science and Technology, v.46, p.1259-1265, 2011.

Ferrari, C. C.; Ribeiro, C. P.; Aguirre, J. M. Secagem por atomização de polpa de amora-preta usando maltodextrina como agente carreador. Brazilian Journal of Food Technology, v.15, p.157-165, 2012. 
Goula, A. M.; Adamopoulos, K. G. A new technique for spray drying orange juice concentrate. Innovative Food Science and Emerging Technologies, v.11, p.342-351, 2010.

Greenspan, L. Humidity fixed points of binary satured aqueuos solutions. Journal of Research of the National of Standards A. Physics and Chemistry, v.81, p.89-96, 1977.

IAL - Instituto Adolfo Lutz. Normas analíticas do Instituto Adolfo Lutz: Métodos químicos e físicos para análise de alimentos. 4.ed. São Paulo: IAL, 2008. 1020p.

IUPAC - International Union of Pure and Applied Chemistry. Reporting Physisorption data for gas solid. Systems with Special Reference to the Determination of Surface Area and Porosity. Pure and Applied Chemistry, v.57, p.603-619, 1985.

Jain, S. K.; Verma, R. C.; Sharma, G. P.; Jain, H. K. Studies on moisture sorption isotherms for osmotically dehydrated papaya cubes and verification of selected models. Journal Food Science Technology, v.47, p.343-346, 2010.

Jaya, S.; Das, H. Effect of maltodextrin, glycerol monostearate and tricalcium phosphate on vaccum dried mango powders properties. Journal of Food Engineering, v.63, p.125-134, 2004.

Kurozawa, L. E.; Park, K. J.; Hubinger, M. D. Effect of maltodextrin and gum arabic on water sorption and glass transition temperature of spray dried chicken meat hydrolysate protein. Journal of Food Engineering, v.91, p.287-296, 2009.

Marques, L. G.; Silveira, A. M.; Freire, J. T. Freeze-drying characteristics of tropical fruits. Drying Technology, v.24, p.457-463, 2006.

Mata, M. E. R. M. C.; Duarte, M. E. M.; Zanini, H. L. H. T. Calor específico e densidade de polpa de cajá (Spondias lutea L.) com diferentes concentrações de sólidos solúveis sob baixas temperaturas. Engenharia Agrícola, v.25, p.488-498, 2005.

Mosquera, L. H.; Moraga, G.; Martínez-Navarrete, N. Effect of maltodextrin on the stability of freeze-dried borojó (Borojoa patinoi Cuatrec.) powder. Journal of Food Engineering, v.97, p.72-78, 2010.
Oliveira, D. M.; Clemente, E.; Costa, J. M. C. Hygroscopic behavior and degree of caking of grugru palm (Acrocomia aculeata) powder. Journal of Food Science and Technology, v.1, p.1-7, 2012.

Oliveira, F. M. N.; Figueiredo, R. M. F.; Queiroz, A. J. M. Análise comparativa de polpas de Pitanga Integral, Formulada e em pó. Revista Brasileira de Produtos Agroindustriais, v.8, p.25-33, 2006.

Pavan, M. A.; Schmidt, S. J.; Feng, H. Water sorption behavior and thermal analysis of freeze-dried, refractance window dried and hot-air dried açaí (Euterpe oleracea Martius) juice. LWT-Food Science and Technology, v.48, p.75-81, 2012.

Silva, A. E.; Silva, L. H. M.; Pena, R. S. Comportamento higroscópico do açaí e cupuaçu em pó. Ciência e Tecnologia de Alimentos, v.4, p.895-901, 2008.

Silva, R. N. G.; Figueiredo, R. M. F. de; Queiroz, A. J. de M.; Galdino, P. O. Armazenamento de umbu-cajá em pó. Ciência Rural, v.35, p.1179-1184, 2005.

Soares, E. B.; Gomes, R. L. F.; Carneiro, J. G. M.; Nascimento, F. N.; Silva, I. C. V.; Costa, J. C. L. Caracterização física e química de frutos de cajazeira. Revista Brasileira de Fruticultura, v.28, p.518-519, 2006.

Strohecker, R; Henning, H. M. Analises de vitaminas: Métodos comprobados. Madrid: Paz Montalvo, 1967. 428p.

Timmermann. E. O. Multilayer sorption parameters: BET or GAB values? Colloids and Surfaces A: Physicochemical and Engineering Aspects, v.220, p.235-260, 2003.

Tonon, R. V., Brabet, C., Hubinger, M. D. Influência da temperatura do ar de secagem e da concentração de agente carreador sobre as propriedades físico-químicas do suco de açaí em pó. Ciência e Tecnologia de Alimentos, v.29, p.444-450, 2009.

Wang, W.; Zhou, W. Characterization of spray-dried soy sauce powders using maltodextrins as carrier. Journal of Food Engineering, v.109, p.399-405. 2012. 